\title{
Motor vehicle unmanned driving technology enhancement
}

\author{
Larisa Zhuravleva \\ Russian State Agrarian University - Moscow Timiryazev Agricultural Academy, \\ Timiryazevskaya str., 49, 127434 Moscow, Russia
}

\begin{abstract}
The relevance of the introduction of unmanned vehicles, the improvement of their control systems is increasing every year due to the ever-increasing volume of cargo transportation. The undoubted advantages of the developed direction are the reduction of the influence of the human factor, management errors, and, consequently, reduction of the number of accidents, as well as reduction of the transportation costs. The research was aimed to develop the algorithmic, programmatic control of an unmanned vehicle when transporting goods along a given route, in particular, when unmanned vehicles move in a convoy behind a pilot vehicle with a given distance along an arbitrary trajectory. The article discusses the control algorithm, technical means of the control system and the technical vision tracking system. The article presents the results of experimental studies of the movement of an unmanned vehicle based on a serial KamAZ 6520 vehicle.
\end{abstract}

\section{Introduction}

The importance of the transport industry can hardly be overestimated. The total freight turnover in Russia is increasing and automobile transport remains the leading mode of transport. The development of transport infrastructure, innovative technologies and technical means comprise the basis for further social-and-economic development, the increase in the volume of foreign and domestic trade, as well as the reduction in the cost of transportation.

Many automotive giant manufacturers and scientific institutions are studying the problem of creating unmanned vehicles capable of moving and performing the assigned technological tasks either without direct human participation or with the minimum human participation in control.

Currently, all world companies conduct the research and work on the creation of unmanned vehicles. The most famous automakers engaged in this area are General Motors, Ford, Mercedes Benz, Volkswagen, Audi, BMW, etc. [1-4].

There is an interesting development of the Volvo company. This is the Safe Road Trains for the Environment (SARTRE) system installed on vehicles moving along the road in a convoy one after another [5]. A convoy of several cars follows a car with a driver, repeating its movement, copying the trajectory with a distance of $6 \mathrm{~m}$.

The control system (New Energy and Industrial Technology Development Organization, NEDO) was also developed to move four trucks in a convoy in Japan to save fuel [6]. 
The most difficult task is to create a so-called intelligent control system enabling a vehicle to perform a task, analyze the external conditions and internal processes in the vehicle systems and make decisions under conditions of uncertainty.

The goal is to develop the algorithmic, programmatic control of an unmanned vehicle for its autonomous operation when driving along a given route under conditions of uncertain road conditions and situations.

A vehicle control system with a set of functions ensuring the unmanned movement along the route using the navigation system and movement in a convoy behind a car with a driver is needed.

\section{Materials and methods}

Considering the movement of a vehicle, the following basic elements of the digital infrastructure should be provided for:

- a linear network of satellite base stations along the road;

- a control center;

- a satellite receiver;

- the communication and information transfer means.

The neural network technology has proven itself in the best way, it is often used in intelligent control systems. For instance, a neural network model that tracks a sequence of images captured during movement is considered in [7].

The work [8] considers the possibility of building trajectories of movement of robotic vehicles when bypassing statically located and moving obstacles. The advantages of such a system are adaptability and high interference immunity.

The authors [9-14, 18-21] considered control systems for mobile robotic means based on the use of semantic and neural networks for pattern recognition, issues of digitalization of the road infrastructure.

Recently, the most widespread are control systems based on fuzzy logic consisting in calculating the optimum required behavior or actions based on the analysis of the current state of the controlled object.

Pursuant to the tasks set, we will present a fuzzy logic unmanned vehicle control system capable of making the real-time decisions. The control system principle is shown in Fig. 1. 


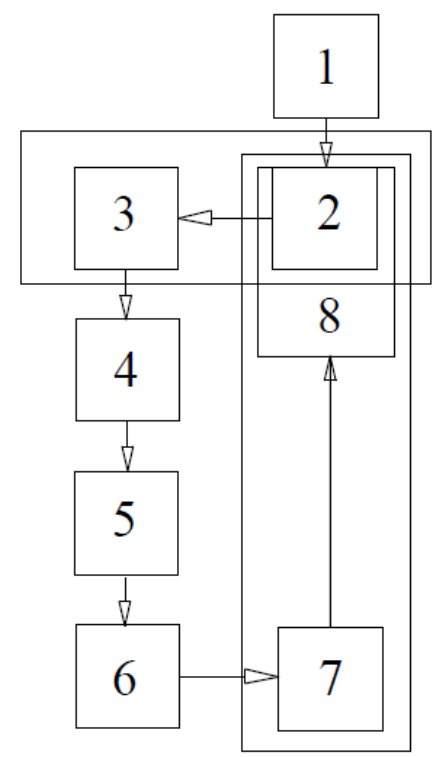

Fig. 1. Control system principle: 1-task, 2-database, 3-control algorithm, 4-actuating mechanism, 5control object, 6-external environment, 7-measuring and information tools, 8- data generalization and decision-making mechanism

Functioning of intellectual control system requires the following:

- technical vision data about the recognizable objects of the environment, their mutual location in relation to an unmanned vehicle;

- data about the state of the internal system of the vehicle, parameters of operation, movement.

The capabilities of the control system should provide for the following modes:

- Mode of direct vehicle control.

- Mode of remote control of a vehicle using a computer.

- Mode of unmanned vehicle control using technical vision.

- Mode of movement in the convoy behind the pilot vehicle.

The simulation diagram of the control system of an unmanned vehicle when driving in a column is shown in Figure 2.

When driving behind a pilot vehicle, in the process of modeling, the coordinates of the location $\mathrm{x}_{0}, \mathrm{y}_{0}$ are determined. The block of motion simulation, considering the position of the unmanned vehicle and the coordinates of the pilot vehicle, determines the direction and distance to it $d$. The task of the fuzzy controller is to determine at each moment of time the linear and angular velocities of the unmanned vehicle.

The input signals for the fuzzy controller are the parameters of the distance $d$ between the unmanned vehicle and the pilot vehicle and the angle $\alpha$ between the direction of movement and the straight line that determines the distance between them.

Since the speed of the pilot vehicle, which sets the mode and trajectory of movement, and relative to which the action of the unmanned vehicle is performed, has a known unknown value, it is necessary for the linear speed controller to proportionally change the speed of movement.

Thus, the controller's task is to determine the required speed of the unmanned vehicle and change it in accordance with the change in the speed of the pilot vehicle, respectively, the task is to timely change or regulate the acceleration. 


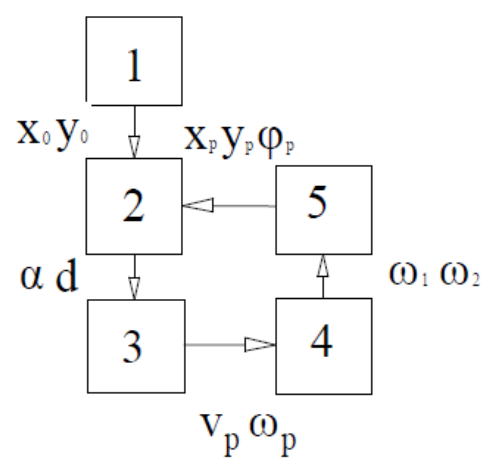

Fig. 2. Simulation scheme of the control system 1-Block of the tracking system of technical vision, analysis of beacon signals, 2-block of motion simulation, 3-fuzzy controller, 4-block of analysis of angular velocities of driving wheels, 5-block of simulation of kinematics and dynamics of a vehicle

If the unmanned vehicle and the pilot vehicle have different speeds and the relative speed has some value, some acceleration value will be determined at the output of the controller.

When turning, the input variable of the controller is the to-the-object direction angle, determined by the built-in vision system. The output variable of the controller is the angular velocity of the unmanned vehicle.

Let us represent the model of motion in the form of a system of equations:

$$
\left\{\begin{array}{l}
\dot{\mathrm{x}}_{0}=\mathrm{v}_{0} \cos \varphi_{0} \\
\dot{\mathrm{y}}_{0}=\mathrm{v}_{0} \sin \varphi_{0}
\end{array}\right.
$$

where $\mathrm{v}_{0}$ is the speed of the pilot vehicle;

$\varphi_{0}$ is the angle of the direction of movement of the pilot vehicle;

$\mathrm{x}_{0}, \mathrm{y}_{0}$ are the coordinates of the position of the pilot vehicle.

Determination of the distance and direction angle is as follows:

$$
\left\{\begin{array}{c}
d=\sqrt{\left(x_{0}-x_{p}\right)^{2}+\left(y_{0}-y_{p}\right)^{2}} \\
\alpha=\operatorname{arctg} 2\left[\left(x_{0}-x_{p}\right),\left(y_{0}-y_{p}\right)\right]-\varphi_{p}
\end{array}\right.
$$

where $\alpha$ is the angle of direction to the object;

$\mathrm{d}$ is the distance between the unmanned vehicle and the pilot vehicle;

$\mathrm{x}_{0}, \mathrm{y}_{0}$ are the coordinates of the position of the pilot vehicle;

$\mathrm{x}_{\mathrm{p}}, \mathrm{y}_{\mathrm{p}}, \varphi_{\mathrm{p}}$ are the coordinates of the position of the unmanned vehicle.

Simulation of the movement of an unmanned vehicle behind a pilot vehicle with movement parameters corresponding to the parameters of the pilot vehicle, repeating its trajectory with a certain specified distance, ensuring traffic safety was carried out in the MatLab program using the Simulink application [15].

For practical confirmation of the reliability of theoretical studies, an experimental model of a system with an unmanned vehicle based on Kamaz 6520 was developed and created for movement along a given trajectory of cargo transportation.

The methodology for studying the characteristics of movement along a curved trajectory in accordance with GOST R 52302-2004 "Road vehicles. Handling and stability. Technical requirements Test methods" [16]. And also GOST R 52280-2004 Trucks. General technical requirements (with Amendment No. 1) [17]. 
The practical study included the movement of vehicles in a convoy one after another and the determination of the characteristics of the movement and trajectory of the unmanned vehicle in the mode of movement behind the pilot vehicle at specified speeds.

The program for controlling the position of servomotors was developed in the MPLab IDE v5.4 program, using the Assembler language.

The set of equipment comprised: a computer control and decision-making unit, a communication system, a set of antennas, a navigation and orientation system.

Following actuators were installed on the unmanned vehicle: linear actuators, electric power steering, and servo drives.

Vision system: SLR 1 light detecting and ranging system; AXIS P1346 video camera and ultrasonic sensors.

The measuring equipment included linear displacement sensors of the actuators and a potentiometer.

\section{Results and discussion}

A number of experiments consisted of determining the trajectory of movement of an unmanned vehicle in the mode of following each other at a certain distance. The value of the distance between the unmanned vehicle and the object in front of the pilot vehicle was taken to be $10 \mathrm{~m}$. The experiments showed the operability of the control system.

The next study was aimed to determine the trajectory of an unmanned vehicle when driving behind a pilot vehicle along an arbitrary trajectory at a speed of $60 \mathrm{~km} / \mathrm{h}$. At the initial moment of time on the remote computer, the system was switched to automatic mode.

After a series of experiments that showed the efficiency and safety of the system, the unmanned vehicle was launched along a given trajectory of the track section.

Graph 3 shows the deviation of the actual trajectory from the one set when making turns. After a number of adjustments, the movement of the unmanned vehicle showed satisfactory results.

After additional experiments with arbitrary movement, the performance of an unmanned vehicle was demonstrated with an acceptable quality of the work process and recommendations were given.

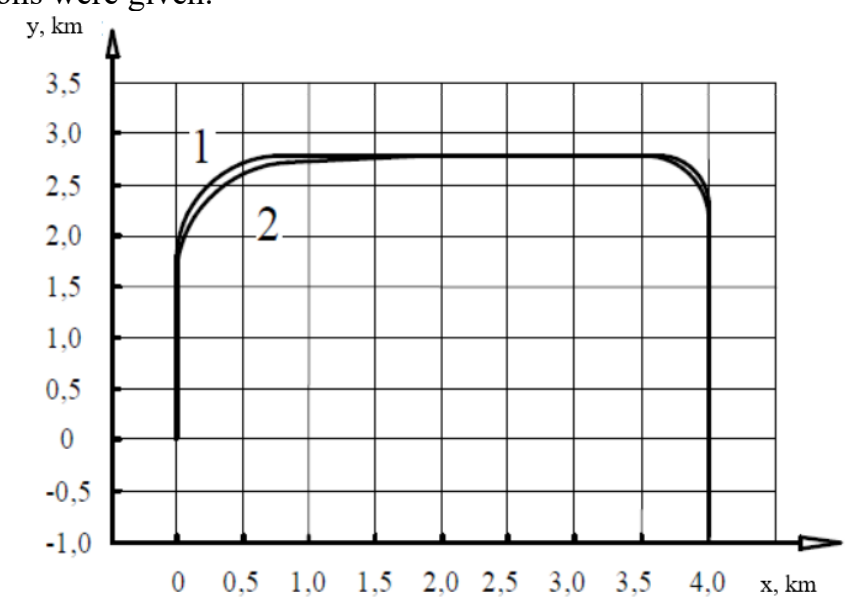

Fig. 3. Movement trajectory: 1-set, 2-actual 


\section{Conclusions}

As a result of the analysis, the prospects of using the fuzzy logic method were substantiated. An algorithm for controlling unmanned vehicles, a mathematical model was developed and computer simulation of movements was carried out.

The equipment was selected and experimental studies were carried out to confirm the theoretical studies.

\section{References}

1. Audi: company website, http://www.audi.com

2. BMW: company website, http://www.bmw.com

3. Google: company website, http://www.google.com

4. Volkswagen: company website, http://www.volkswagenag.com

5. Volvo Trucks - self-driving road caravan vehicles, http:/interdalnoboy.com

6. Japan launches a road train with robotic trucks on the road, http:/www. nanonewsnet.ru

7. Fundamentals of artificial neural networks, http://www.intuit.ru

8. V. M. Lokhin, I. M. Makarov, S.V. Manko, M. P. Romanov, Extreme robotics, 73 (1999)

9. V. V. Kruglov, Fuzzy logic and artificial neural networks (2001)

10. Zh.L. Loryer, Systems of artificial intelligence (1991)

11. V. M. Lokhin, M. P. Romanov, Extreme robotics, 1 (2014)

12. D.A. Pospelova. Models and methods. Artificial Intelligence (1990)

13. Neurocybernetics, neuroinformatics, neurocomputers, http://www.bigrouplabs.ru.

14. Fundamentals of artificial neural networks, http://www.intuit.ru.

15. Matlab: Company website, http://matlab.ru

16. GOST R 52302- 2004 "Road vehicles. Handling and stability. Technical requirements Test methods".

17. GOST R 52280-2004 Trucks. General requirements (With Amendment 1)

18. T. Peter, Int. J. of Applied Mathematics and Computer Sciences, 3 (2012)

19. J. Hu, D. Zhao, F. Zhu, Proc. 14th International IEEE Conference on Intelligent Transportation Systems (ITSC) (2011)

20. A.I. Diveev and E.A. Sofronova, Proceedings of European Control Conference (ECC2013) (2013)

21. G.B. Castro, J.C. Martini, A. Hirakawa, Proc. of 17th International Conference on Intelligent Transportation Systems (2014) 